\title{
Myocardial perfusion imaging for cardiac allograft vasculopathy assessment: Evidence grows, but questions remain
}

\author{
Deepak Acharya, MD, MSPH, ${ }^{a}$ and Indranee Rajapreyar, $\mathrm{MD}^{\mathrm{a}}$ \\ a Section of Advanced Heart Failure Transplantation, and Mechanical Circulatory Support, \\ University of Alabama at Birmingham, Birmingham, AL
}

Received Oct 21, 2017; accepted Oct 23, 2017

doi: $10.1007 / \mathrm{s} 12350-017-1116-4$

\section{See related article, pp. 845-852}

Cardiac allograft vasculopathy (CAV) is a leading cause of long-term mortality after heart transplantation. CAV can present insidiously because of denervation of the transplanted heart, and significant heart failure or sudden death can be the first presentation. The median survival of patients transplanted between 1992 and 2001 is 10.5 years. CAV develops in one-third of heart transplant survivors at 5 years and in one-half at 10 years post-transplant, and is responsible for at least $12.8 \%$ of deaths at 10 years. ${ }^{1}$ Risk factor modification and early therapy with mammalian target of rapamycin (mTOR) inhibitors such as sirolimus may attenuate CAV progression. ${ }^{2,3}$ Percutaneous coronary intervention (PCI) or coronary artery bypass grafting (CABG) are useful for focal lesions but suboptimal in advanced disease because of diffuse disease, poor targets, and high restenosis rates. Retransplantation is effective, but raises ethical concerns, is severely hampered by organ shortage, and requires planning and waiting during which time clinical events can occur. Therefore, early detection and formulation of strategies to prevent or treat CAV is important in optimizing overall outcomes after heart transplantation.

Historically, coronary angiography (CAG) has been the preferred modality for CAV screening. However, there are many limitations of $\mathrm{CAG}$, including

Reprint requests: Deepak Acharya, MD, MSPH, Section of Advanced Heart Failure, Transplantation, and Mechanical Circulatory Support, University of Alabama at Birmingham, 1900 University Blvd, THT 321, Birmingham, 35294; dacharya@uab.edu

J Nucl Cardiol 2019;26:853-6.

$1071-3581 / \$ 34.00$

Copyright (C 2017 American Society of Nuclear Cardiology. invasiveness, risk of contrast nephropathy, and most importantly relative insensitivity to detect CAV given the diffuse nature of disease, inability to directly assess the intima, lack of a reference segment and microvascular involvement. Approximately 50\% of heart transplant recipients develop renal dysfunction at 5 years post-transplant, and routine use of surveillance angiography becomes challenging. ${ }^{1}$ Patients with reportedly normal CAGs who died from graft failure have a high prevalence of significant CAV on autopsy. ${ }^{4}$ As a result, several other anatomic and functional, invasive and non-invasive modalities, including dobutamine stress echocardiography (DSE), myocardial perfusion imaging (MPI), positron emission tomography (PET), computed tomography (CT), Magnetic Resonance Imaging (MRI), intravascular ultrasound (IVUS), and optical coherence tomography (OCT), have been evaluated in $\mathrm{CAV} .^{5}$ The majority of these studies have been single-center, observational, with heterogeneous populations, differing methodologies, and only a few have evaluated long-term outcomes.

In this issue of the Journal, Veenis and colleagues report on the long-term prognostic value of exercise/dobutamine 99mTc-tetrofosmin SPECT MPI performed at a mean time of 7.4 years post heart transplant in 166 patients between 1992 and 1998. ${ }^{6}$ The short-term results, published in 2002, found that routine clinical variables did not predict cardiac death, but peak rate-pressure product and abnormal myocardial perfusion were significant predictors of cardiac death at a median follow-up of 2.5 years post MPI. ${ }^{7}$ This study extended the follow-up to mean 9.5 years and concluded that patients with normal SPECT had lower major cardiac events up to 1 year after testing, lower cardiac mortality up to 2 years after testing, and lower all-cause mortality up to 5 years after testing compared to patients with abnormal SPECT. By multivariate analysis, the presence of a reversible perfusion defect was a predictor 
of cardiac mortality, cardiac events, and all-cause mortality for up to 5 years. An abnormal SPECT (fixed and/ or reversible defect) was predictive of cardiac events and cardiac mortality for 2 years, and predictive of overall mortality for up to 5 years. The authors report a warranty period of 5 years after a normal MPI and support the role of SPECT MPI to avoid coronary angiography in some cases.

Previous studies of myocardial perfusion imaging to detect CAV have reported varied results, due to multiple factors including disparate definitions of angiographic CAD, variable time interval between transplantation and testing, diagnostic vs. prognostic aims, choices of tracer agents and stress modalities. ${ }^{5}$ Furthermore, most have compared MPI to CAG, which has limitations because of diffuse vessel involvement with lack of reference segment for comparison and presence of small vessel disease. A few salient points are highlighted. Carlsen et al. evaluated 66 patients with primarily dipyridamole myocardial scintigraphy with $99 \mathrm{mTc}$-Tetrofosmin or 99mTc-sestamibi and CAG annually for mean follow-up of 5.6 years. The negative predictive value of a normal SPECT for excluding CAD requiring revascularization was $98 \%{ }^{8}$ Ciliberto and colleagues evaluated 78 patients with rest echocardiography, dipyridamole $99 \mathrm{~m}$ Tc sestamibi SPECT, and CAG and found that a combination of normal echo and SPECT had 100\% negative predictive value (NPV) for significant CAV and 82\% NPV for any CAV by CAG. Abnormal echo and SPECT were associated with $60 \%$ sensitivity for any CAV and $100 \%$ sensitivity for severe CAV. Discordant echo and MIBI results were associated with intermediate CAV rates. An initial non-invasive strategy with selective angiogram in patients with positive or discordant noninvasive tests was cost-effective. ${ }^{9}$ Another cohort of 110 patients who underwent gated exercise \pm dipyridamole SPECT with Tl-201 or Tc-99-sestamibi at a mean of 66 months after transplant and CAG within a month of SPECT were followed for an average of 57 months. Stress perfusion defect $>3$ segments by SPECT was an independent predictor of death or retransplantation, and provided incremental information to CAV by CAG on multivariate analysis. Among patients who had normal functional and perfusion gated SPECT parameters (normal stress perfusion, $\mathrm{EF} \geq 50$, and $\mathrm{ESV} \leq 35 \mathrm{ml}$ ), risk of cardiac death or retransplantation was low, with the first event occurring 27 months after SPECT. The sensitivity, specificity, positive predictive value (PPV), and NPV for identifying severe CAV were $84,70,37$, and $96 \%$. For detection of any CAV, sensitivity was $63 \%$ and NPV was $75 \% .{ }^{10}$ Due to limited coronary flow reserve and diffuse involvement of coronary arterial bed, vasodilators like dipyridamole and adenosine may have limited sensitivity to detect early CAV. ${ }^{11} \mathrm{Wu}$ and colleagues evaluated 47 patients with echo, CAG, and dobutamine 201-Tl SPECT and found that sensitivity, specificity, PPV and NPV of SPECT for detecting significant CAV were 89, 71, 42, and 96\%. Large reversible perfusion defects indicated significant $\mathrm{CAD}$ and predicted cardiac death. Lung Heart Ratio $\geq 0.37$ during stress provided incremental information to perfusion defects. ${ }^{12}$ Another report of 104 patients who underwent exercise 99m Tc-Tetrofosmin SPECT, those with inhomogeneous stress (but not rest) perfusion despite no significant angiographic CAV had a higher risk of developing allograft dysfunction but not angiographic $\mathrm{CAV}$ at a follow-up of almost 10 years, highlighting the potential of SPECT to detect microvascular and endothelial dysfunction not detectable on angiography. ${ }^{13}$

Particular strengths of the present study include the relatively large population, uniform testing techniques, assessment of hard clinical endpoints, and long followup duration with outcome data available on all patients. The major limitation that reduces generalizability is the lack of data on angiographic findings and subsequent management. The authors report that per their current protocol, CAG is performed in the first and fourth year after transplant, and annual SPECT from the sixth year from transplant, with CAG reserved for patients with clinical indications or abnormal SPECT. During the early part of their experience, when the prognostic significance of SPECT was not clear, CAG was also performed at other time points. Management decisions such as PCI, CABG, or retransplant that influenced the reported outcomes were not made only based on the SPECT data but involved CAGs and other testing. Furthermore, it is not clear that all patients who underwent transplantation and were followed at the authors' institution during the study period were enrolled in the study, and patients were enrolled at a mean of 7.4 years after transplant, leading to some selection and survivorship bias and pre-existing knowledge of the presence or absence of CAV in individual patients. SPECT was performed annually after the sixth year, even in patients who had the single normal study-analyzed SPECT and there may have been additional opportunities to detect new CAV and alter management. Therefore, while this study demonstrates higher overall survival up to 5 years after a normal SPECT, it does not provide specific information on diagnostic accuracy of a single SPECT for CAV detection long term, risk stratification, or guidelines for management, in particular avoidance of angiography. We don't know what proportion of patients with normal SPECT perfusion had $\mathrm{CAG}$, angiographic $\mathrm{CAD}$ or subsequent revascularization. It is also important to note that although patients with normal SPECT had better outcomes than those with abnormal SPECT, they were not a low-risk population, 
with approximately $20 \%$ overall mortality and $10 \%$ cardiac mortality at 2 years. Clinical end points that could have provided incremental prognostic information, such as revascularization or retransplantation, were not included. Therefore, the implied 5-year warranty of a normal SPECT is a limited warranty, with some fine print.

A few other study findings deserve comment. First, consistent with other studies, reversible defects had more prognostic significance than fixed defects, indicating viable myocardium in jeopardy. Second, approximately $30 \%$ of patients did not achieve target heart rate. SPECT in heart transplantation can be performed with exercise, dobutamine, and vasodilators. Heart transplant patients may have chronotropic incompetence, and rates of permanent pacemaker implantation range from $2-24 \% .{ }^{14}$ Heart rate response should be a consideration in selecting the stress modality. Finally, only patients $>2$ years post-transplant were enrolled. While late CAV is an important contributor to mortality, CAV can also progress rapidly early after transplant, and the benefit of tailored immunosuppression for $\mathrm{CAV}$ is most effective when initiated within the first 2 years after transplantation. ${ }^{3,15}$ The role of SPECT in early CAV diagnosis remains uncertain.

There is a distinction between the diagnostic and prognostic value of tests that screen for CAV, and the appropriate test depends on the purpose of the examination. If the purpose of the test is to detect early nonflow-limiting CAV in order to make decisions regarding medical management, for example early initiation of mTOR inhibitors, SPECT may not be sufficiently accurate or sensitive, but the same is true about dobutamine echocardiogram or coronary angiography. In this setting IVUS, OCT, or CTA may be more appropriate in imaging the vessel wall in addition to the lumen. As an example, CAV that is angiographically silent but progressive by IVUS, defined as $\geq 0.5 \mathrm{~mm}$ intimal thickening in the first year post-transplant, was a predictor of mortality and future development of angiographic CAV. ${ }^{16}$ PET, while expensive and not widely available, may also quantify myocardial blood flow and can demonstrate early CAV in patients who have angiographically normal coronary arteries. ${ }^{17}$ If the purpose of the test is to evaluate for severe CAV which would mandate angiography and revascularization, gated SPECT appears to be sufficiently sensitive, and the high negative predictive value of a normal test may safely allow delay or decrease in frequency of angiograms in the short term. If the purpose is to assess overall prognosis, for example for clinical trials, gated SPECT integrates macrovascular, microvascular, and functional data and provide information not available from CAG. The low PPV and specificity of SPECT studies that have used CAG as the gold standard should be interpreted with caution, since perfusion defects on SPECT may reflect true abnormalities that may have prognostic value but are undetected by CAG. Dobutamine echo, PET and IVUS have also shown prognostic value in multiple studies, whereas MRI, CTA, and OCT have scarce prognostic data. An individualized approach incorporating clinical variables (donor and recipient characteristics), renal function, rejection history, and the presence of donor specific antibodies can be used to make decisions regarding invasive vs non-invasive CAV surveillance long term if CAG with intravascular imaging within the first 3-5 years after transplant does not reveal CAV.

In summary, this study strengthens conclusions from prior studies, and provides additional insights into factors that affects long-term prognosis in CAV. SPECT is an attractive modality for CAV assessment given widespread availability, expertise, safety, and relatively low cost. Aggregate data from all the available studies show that a completely normal SPECT is reassuring in terms of clinical endpoints. The clinical implication is that while SPECT may not detect early $\mathrm{CAV}$, it can be used to safely exclude severe disease and has a role in decreasing or delaying angiography, particularly in patients who have had previously normal CAGs or those who are already being treated with all available medical therapies. While it is clear that angiography is less than ideal, questions remain regarding the best screening technique. Prospective, multicenter, head-to-head comparisons of the multiple invasive and non-invasive modalities have not yet been performed to guide the best approach and establish new gold standards. Until there is more robust evidence, there is unlikely to be widespread adoption of one particular technique, and the most appropriate test depends on study goals, local expertise, availability, and protocols.

\section{Disclosure}

None.

\section{References}

1. Lund LH, Khush KK, Cherikh WS, et al. The Registry of the International Society for Heart and Lung Transplantation: thirtyfourth Adult Heart Transplantation Report-2017; Focus Theme: Allograft ischemic time. J Heart Lung Transplant 2017;36:103746.

2. Mancini D, Pinney S, Burkhoff D, et al. Use of rapamycin slows progression of cardiac transplantation vasculopathy. Circulation 2003;108:48-53. 
3. Raichlin E, Bae JH, Khalpey Z, et al. Conversion to sirolimus as primary immunosuppression attenuates the progression of allograft vasculopathy after cardiac transplantation. Circulation 2007;116:2726-33.

4. Clague JR, Cox ID, Murday AJ, et al. Low clinical utility of routine angiographic surveillance in the detection and management of cardiac allograft vasculopathy in transplant recipients. Clin Cardiol 2001;24:459-62.

5. Payne GA, Hage FG, Acharya D. Transplant allograft vasculopathy: Role of multimodality imaging in surveillance and diagnosis. J Nucl Cardiol 2016;23:713-27.

6. Veenis JF, Boiten HJ, van den Berge JC, et al. Prediction of longterm ( $>10$ year) cardiovascular outcomes in heart transplant recipients: Value of stress technetium-99m tetrofosmin myocardial perfusion imaging. J Nucl Cardiol. https://doi.org/10.1007/ s12350-017-1089-3.

7. Elhendy A, van Domburg RT, Vantrimpont P, et al. Prediction of mortality in heart transplant recipients by stress technetium- $99 \mathrm{~m}$ tetrofosmin myocardial perfusion imaging. Am J Cardiol 2002;89:964-68.

8. Carlsen J, Toft JC, Mortensen SA, et al. Myocardial perfusion scintigraphy as a screening method for significant coronary artery stenosis in cardiac transplant recipients. J Heart Lung Transplant 2000; $19: 873-78$

9. Ciliberto GR, Ruffini L, Mangiavacchi M, et al. Resting echocardiography and quantitative dipyridamole technetium- $-99 \mathrm{~m}$ sestamibi tomography in the identification of cardiac allograft vasculopathy and the prediction of long-term prognosis after heart transplantation. Eur Heart J 2001;22:964-71.
10. Manrique A, Bernard M, Hitzel A, et al. Diagnostic and prognostic value of myocardial perfusion gated SPECT in orthotopic heart transplant recipients. J Nucl Cardiol 2010;17:197-206.

11. Thompson D, Koster MJ, Wagner RH, et al. Single photon emission computed tomography myocardial perfusion imaging to detect cardiac allograft vasculopathy. Eur Heart J Cardiovasc Imaging 2012;13:271-75.

12. Wu YW, Yen RF, Lee CM, et al. Diagnostic and prognostic value of dobutamine thallium-201 single-photon emission computed tomography after heart transplantation. J Heart Lung Transplant 2005;24:544-50.

13. Wenning C, Vrachimis A, Dell Aquila A, et al. Inhomogeneous myocardial stress perfusion in SPECT studies predicts future allograft dysfunction in heart transplant recipients. EJNMMI Res 2015;5:51.

14. Mallidi HR, Bates M. Pacemaker use following heart transplantation. Ochsner J 2017;17:20-24.

15. Matsuo Y, Cassar A, Yoshino S, et al. Attenuation of cardiac allograft vasculopathy by sirolimus: Relationship to time interval after heart transplantation. J Heart Lung Transplant 2013;32:78491.

16. Tuzcu EM, Kapadia SR, Sachar R, et al. Intravascular ultrasound evidence of angiographically silent progression in coronary atherosclerosis predicts long-term morbidity and mortality after cardiac transplantation. J Am Coll Cardiol 2005;45:1538-42.

17. Wu YW, Chen YH, Wang SS, et al. PET assessment of myocardial perfusion reserve inversely correlates with intravascular ultrasound findings in angiographically normal cardiac transplant recipients. J Nucl Med 2010;51:906-12. 